\title{
Avaliação da ação de um agente dessensibilizante na remineralização dos túbulos dentinários antes e após serem submetidos a clareamento dental: estudo in vitro
}

Evaluation of the action of a desensibilizing agent in the remineralization of dental tubes before and after being subjected to dental clarification: in vitro study

Evaluación de la actuación de un agente desensibilizante en la remineralización de tubos dentales antes y después de ser sujeto a clarificación dental: estudio in vitro

\author{
Sofia Hiluey de Aguiar Leite \\ ORCID: https://orcid.org/0000-0002-2316-2913 \\ Universidade Estadual da Paraíba, Brasil \\ E-mail: sofiahilueyleite@gmail.com \\ Alieny Cristina Duarte Ferreira \\ ORCID: https://orcid.org/0000-0003-0370-3177 \\ Universidade Estadual da Paraíba, Brasil \\ E-mail: alienycris@hotmail.com \\ Ana Luzia Araújo Batista \\ ORCID: https://orcid.org/0000-0002-7780-060X \\ Universidade Estadual da Paraíba, Brasil \\ E-mail: analuziabatista@globo.com \\ José de Alencar Fernandes Neto \\ ORCID: https://orcid.org/0000-0003-3711-6966 \\ Universidade Estadual da Paraíba, Brasil \\ E-mail: jneto411@gmail.com \\ Tiago Almeida de Oliveira \\ ORCID: https://orcid.org/0000-0003-4147-7721 \\ Universidade Estadual da Paraíba, Brasil \\ E-mail: tadolive84@gmail.com \\ Maria Helena Chaves de Vasconcelos Catão \\ ORCID: https://orcid.org/0000-0001-7681-3225 \\ Universidade Estadual da Paraíba, Brasil \\ E-mail: mhelenact@zipmail.com.br
}

\begin{abstract}
Resumo
O objetivo deste estudo foi avaliar, in vitro, através de análise de microdureza e rugosidade a ação de um agente dessensibilizante sobre o esmalte dentário antes e/ou depois de serem submetidos a clareamento dental. Foram utilizados 40 incisivos bovinos para a obtenção de 40 fragmentos de esmalte. As amostras foram divididas em 5 grupos experimentais $(n=8)$ : controle positivo - sem tratamento clareador, controle negativo - agente clareador, G1 agente clareador + dessensibilizante, G2 - agente dessensibilizante + clareador, G3 - agente dessensibilizante + clareador + dessensibilizante. Foram realizadas 3 aplicações, 8 minutos cada, do gel clareador e 1 aplicação de 10 minutos de dessensibilizante. Os tratamentos seguiram sequências de acordo com os grupos experimentais. Antes da realização dos tratamentos, as amostras passaram por 3 leituras de rugosidade superficial e microdureza, as quais foram repetidas no momento posterior aos tratamentos. Os dados foram tabulados e submetidos as análises estatísticas ANOVA, Tukey, Wilcoxon e Teste de Dunnett, considerando os fatores tratamento e tempo. A análise estatística dos dados revelou que, mesmo existindo uma diminuição da rugosidade, não houve diferença estatisticamente significante antes e após a aplicação do agente dessensibilizante e gel clareador.
\end{abstract}

Palavras-Chave: Clareamento dental; Sensibilidade da dentina; Clareadores dentários; Dessensibilizantes dentinários.

\begin{abstract}
The aim of this study was to evaluate, in vitro, through microhardness and roughness analysis, the action of a desensitizing agent on tooth enamel before and/or after being subjected to tooth whitening. Forty bovine incisors were used to obtain 40 enamel fragments. The samples were divided into 5 experimental groups $(\mathrm{n}=8)$ : positive control without bleaching treatment, negative control - bleaching agent, G1 - bleaching + desensitizing agent, G2 desensitizing + bleaching agent, G3 - desensitizing + bleaching + desensitizing agent. Three applications were made, 8 minutes each, of the bleaching gel and 1 application of 10 minutes of desensitizing. The treatments followed sequences according to the experimental groups. Before the treatments were carried out, the samples went through 3
\end{abstract}


readings of surface roughness and microhardness, which were repeated after the treatments. The data were tabulated and submitted to statistical analysis ANOVA, Tukey, Wilcoxon and Dunnett's test, considering the treatment and time factors. The statistical analysis of the data revealed that, although there was a decrease in roughness, there was no statistically significant difference before and after the application of the desensitizing agent and whitening gel.

Key Words: Tooth whitening; Dentin sensitivity; Tooth whitening; Dentin desensitizers.

\section{Resumen}

El objetivo de este estudio fue evaluar, in vitro, mediante análisis de microdureza y rugosidad, la acción de un agente desensibilizante sobre el esmalte dental antes y/o después de ser sometido al blanqueamiento dental. Se utilizaron cuarenta incisivos bovinos para obtener 40 fragmentos de esmalte. Las muestras se dividieron en 5 grupos experimentales $(\mathrm{n}=8)$ : control positivo - sin tratamiento blanqueador, control negativo - blanqueador, G1 blanqueador + desensibilizante, G2 - desensibilizante + blanqueador, G3 - desensibilizante + blanqueador + desensibilizante. Se realizaron tres aplicaciones, de 8 minutos cada una, del gel blanqueador y 1 aplicación de 10 minutos de desensibilizante. Los tratamientos siguieron secuencias según los grupos experimentales. Antes de realizar los tratamientos, las muestras pasaron por 3 lecturas de rugosidad superficial y microdureza, que se repitieron después de los tratamientos. Los datos fueron tabulados y sometidos a análisis estadístico ANOVA, Tukey, Wilcoxon y prueba de Dunnett, considerando los factores de tratamiento y tiempo. El análisis estadístico de los datos reveló que, aunque hubo una disminución de la rugosidad, no hubo diferencia estadísticamente significativa antes y después de la aplicación del agente desensibilizante y el gel blanqueador.

Palabras Clave: Blanqueamiento dental; Sensibilidad dentinaria; Blanqueamiento de dientes; Desensibilizadores de dentina.

\section{Introdução}

A dentina e a polpa são tecidos que possuem a mesma origem, provenientes da mesma estrutura embriológica, os odontoblastos, e permanecem intimamente relacionados durante toda a vida funcional do dente sendo tratado como uma estrutura integrada, o complexo dentino-pulpar. A principal característica desse complexo é a presença de túbulos da dentina até a polpa, com prolongamentos odontoblásticos e fluídos comunicando as duas estruturas de forma que todas as injúrias impostas à dentina repercutem instantaneamente ao tecido pulpar (Hebling, Ribeiro, \& Costa, 2010).

A sensibilidade dentinária é compreendida como uma dor de curta duração, aguda e súbita, podendo ser proveniente de inúmeros fatores como escovação inadequada, erosão por dieta alimentar ácida, terapia periodontal, tratamento restaurador incorreto, recessão gengival, uso de abrasivos, higiene oral deficiente, contato oclusal prematuro, trauma oclusal e exposição a um agente ácido (Rocha, Prado, Simão, Lima, \& Gusman, 2016). O diagnóstico da sensibilidade dentária não é simples pois pode estar associado a vários fatores, no entanto, nos casos em que está relacionada com o clareamento dental, esta é diagnosticada facilmente pois a sintomatologia dolorosa inicia logo após as sessões de clareamento (Crepaldi, \& Marques, 2018; Douglas-de-Oliveira, de Paiva, \& Cota, 2017; Gillam, 2013).

Os mecanismos envolvidos na sensibilidade dentinária após o clareamento não foram totalmente esclarecidos. A teoria hidrodinâmica sobre a hipersensibilidade postula que a dor se dá pela movimentação de fluídos dentro dos túbulos dentinários após a dentina ser exposta ao meio bucal por algum motivo, estimulando as terminações nervosas pulpares presentes na figura dos prolongamentos odontoblásticos no interior dos túbulos (Brännström, Lindén, \& Åström, 1967; Rebelo, Loureiro, Ferreira, Paula, \& Carrilho, 2011; Gomes, Filho, Penelas, \& Fonseca, 2015).

No clareamento dental a exposição da dentina se dá pela ação dos agentes clareadores, peróxido de carbamida (10 a 16\%) e peróxido de hidrogênio (35 a 37\%), que atuam de forma semelhante, quebrando as cadeias moleculares dos cromóforos, pigmentos escuros responsáveis pelo manchamento dental, e induzindo sua eliminação da dentina através de difusão resultando em uma sensibilidade dentinária (Pintado-Palomino, Peitl Filho, Zanotto, \& Tirapelli, 2015) que pode ser reparada ou reduzida por diversos mecanismos fisiológicos incluindo a precipitação de proteínas e cristais intratubulares, formação de dentina esclerótica e reparadora, e invasão dos túbulos por bactérias ou por proteínas plasmáticas. Contudo, em alguns indivíduos esta sensibilidade não reduz e persiste, necessitando de tratamento (Pashley, 1986).

O tratamento para hipersensibilidade pode ser realizado por meio de mecanismos da inibição da propagação do 
estímulo doloroso utilizando produtos com sais de potássio, através da oclusão dos túbulos dentinários utilizando materiais restauradores, laser ou agentes remineralizadores, como também através de uma abordagem combinada de administração sistêmica de analgésicos / antiinflamatórios e aplicação tópica de um dessensibilizante (Araújo et al,. 2012). A primeira linha de tratamento para a hipersensibilidade são os dessensibilizantes, agentes remineralizadores que atuam promovendo a obliteração dos túbulos dentinários e também reduzindo a excitabilidade da terminação nervosa intradental, diminuindo a frequência e intensidade da sensibilidade sem afetar a eficiência do procedimento clareador (Markowitz, 2010; Cerqueira, Hofstaetter, Rezende, Martins, Loguercio, Reis, \& Kossatz, 2013).

A aplicação tópica dos dessensibilizantes previamente ou imediatamente após sessões de clareamento têm sido recomendada para evitar ou minimizar desconforto dos pacientes com a sensibilidade dentária (Públio, D'Arce, Ambrosano, Aguiar, Lovadino, Paulillo, \& Lima, 2015; Goncalves, \& Santos, 2017). Portanto, diante desse cenário de utilização cada vez mais frequente do dessensibilizante como alternativa de tratamento a hipersensibilidade por clareamento é importante conhecer ainda mais sobre as implicações do seu uso. O objetivo deste estudo foi avaliar, in vitro, através de análise de microdureza e rugosidade a ação de um agente dessensibilizante sobre o esmalte dentário de dentes bovinos, antes e/ou depois de serem submetidos a clareamento dental.

\section{Metodologia}

O presente estudo é do tipo experimental in vitro, composto por uma amostra de 40 dentes incisivos bovinos. As variáveis estudadas foram rugosidade superficial e microdureza e os fatores de variação compreenderam o tratamento clareador com peróxido de hidrogênio a 35\% (Kit Lase Peroxide Flex - DMC) e o tratamento dessensibilizante remineralizador com o gel dessensibilizante duo desense - nitrato de potássio a 5\% e fluoreto de sódio a 2\% (Desensibilize KF0,2\% - FGM).

O preparo da amostra compreendeu o seccionamento na junção amelo-cementaria, separando a parte coronaria das raízes, perpendicularmente em relação ao longo eixo do dente com auxílio de um broca (Carbide Fg 702 19mm Prima Angelus), em seguida foram realizados cortes longitudinais e tranversais na coroa com a cortadeira de precisão (Isomet 1000Buehler Ltda, llinois, USA), excluindo a porção incisal, cervical e proximal, utilizando apenas para este estudo a porção central da coroa bovina ( $4 \mathrm{~mm} 2$ de dimensão), obtendo-se 40 espécimes de esmalte e dentina $(4 \times 4 \times 3 \mathrm{~mm})$ conferindo seu tamanho com um auxílio de um paquímetro, as superfícies incisais, cervicais, proximais, vestibulares e linguais dos blocos planificados em Politriz giratória (APL-4, Arotec, Cotia, SP. Brasil), com lixas de carbeto de silício nas granulações 320, 600 e 1200 (Norton, Vinhedo, SP, Brasil) com 30 s para cada granulação, sob refrigeração constante. Após o uso das lixas, os corpos de prova foram polidos em feltros com pastas de polimento com granulações de 1 a $0,3 \mu \mathrm{m}$ para possibilitar a obtenção de uma superfície lisa, regular, plana e polida, logo após foram fixados dentro de um tubo de PVC preenchido com resina acrílica (VIPI Flash), enumerados, imersos em água destilada e armazenados (Figura 1). 
Figura 1: a e b - cortadeira de precisão e dente acoplado a matriz de acrílico para confecção dos corpos de prova; c espécimes confeccionados com resina acrílica e cano PVC.

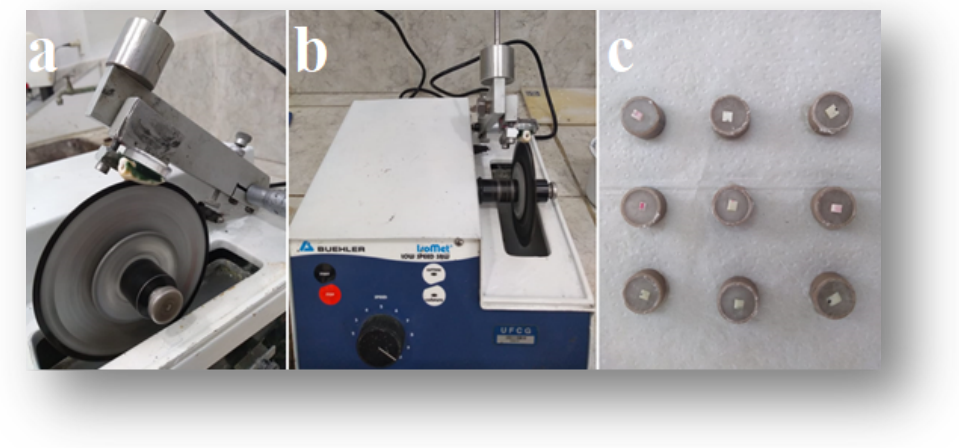

Fonte: Autores.

As amostras foram divididas aleatoriamente em cinco grupos, cada um contendo oito amostras de substratos de esmalte e dentina, grupo controle positivo, grupo controle negativo e três grupos experimentais. O grupo controle positivo (GP) foi exposto apenas a saliva artificial e o grupo controle negativo (GN) exposto apenas ao tratamento clareador. Os grupos experimentais foram divididos em: grupo 1 (G1), exposto ao tratamento clareador e em seguida ao tratamento dessensibilizante; grupo 2 (G2): exposto ao tratamento dessensibilizante e em seguida ao tratamento clareador; grupo 3 (G3): exposto ao tratamento dessensibilizante, em seguida ao tratamento clareador e logo após o tratamento dessensibilizante.

O tratamento clareador seguiu as recomendações do fabricante e foi realizado em uma sessão 3 aplicações de peróxido de hidrogênio a $35 \%$ por 8 minutos, seguido de lavagem com água corrente por 30 segundos e secas com papel absorvente (figura 2). O tratamento dessensibilizante também seguiu as recomendações do fabricante e o gel dessensibilizante duo desense foi aplicado na superfície das amostras por 10 minutos (figura 3). Após os tratamentos as amostras foram lavadas com água corrente durante 30 segundos, secas com papel absorvente e novamente armazenadas em água destilada.

Figura 2: a- peróxido de hidrogênio 35\% (Kit Lase Peroxide Flex - DMC); b- Aplicação de agente clareador no G1.

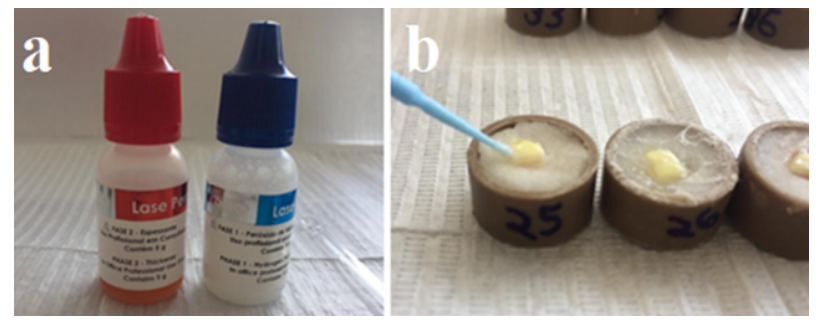

Fonte: Autores.

Figura 3: a - gel dessensibilizante duo desense - nitrato de potássio a 5\% e fluoreto de sódio a 2\% (Desensibilize KF0,2\% - FGM); b - Aplicação do gel dessensibilizante no grupo 1.

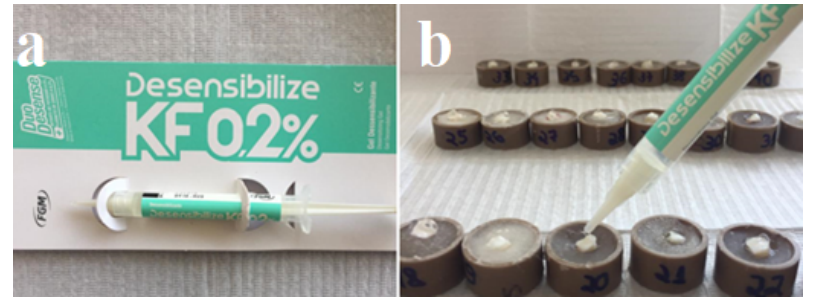

Fonte: Autores. 
Os testes de rugosidade e microdureza foram realizados antes e após os tratamentos com o agente clareador e o agente dessensibilizante nos grupos controle negativo e nos grupos experimentais e, também, antes e após o armazenamento com saliva artificial do grupo controle positivo.

A análise de microdureza utilizou um Microdurômetro (Microhardness Tester FM-700, Future-tech) (figura 4) com penetrador diamantado piramidal tipo Knoop, programado para aplicação de 100gf de carga por 15 segundos. Foram realizadas 3 indentações em áreas distintas na superfície de cada espécime e realizada a média entre elas (figura 5). O ensaio foi realizado antes e após a experimentação dos grupos.

Figura 4: a - Microdurômetro; b - Momento de realização da indentação.

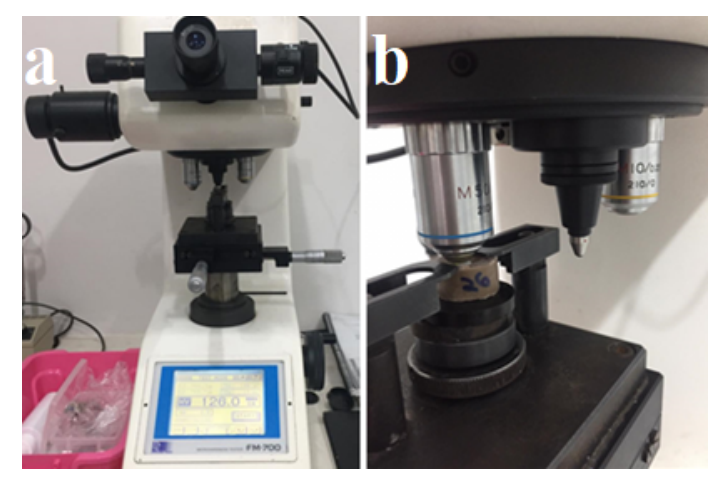

Fonte: Autores.

Figura 5: Etapas do ensaio de microdureza.

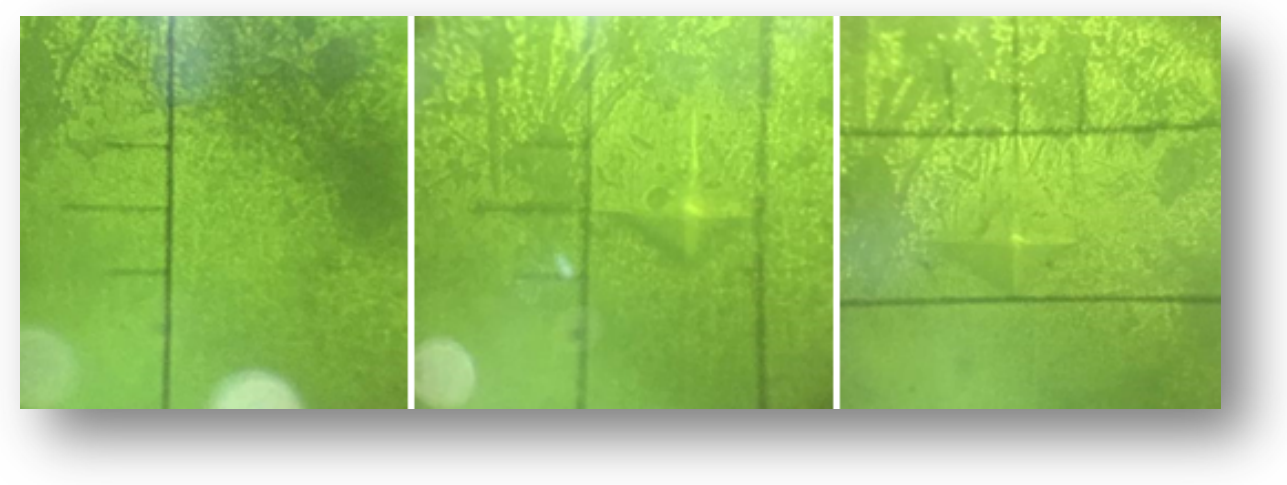

Fonte: Autores.

A análise da rugosidade superficial dos espécimes foi aferida, através do microscópio óptico digital (HIRAX 7700) (figura 6), com aumento de 140X e auxílio do software Gwyddion. Os espécimes foram escolhidos aleatoriamente, resultando em 4 espécimes por grupo (50\% da amostragem), e submetidos a microscopia óptica. Foram capturadas 2 fotomicrografias em áreas distintas de cada amostra e, em seguida, a topografia das imagens foram analisadas através do Software Gwyddion, obtendo-se a Rugosidade ( $\mathrm{Ra})$ de cada espécime analisado e também a média por grupo. 
Figura 6: Microscópio óptico e amostra.

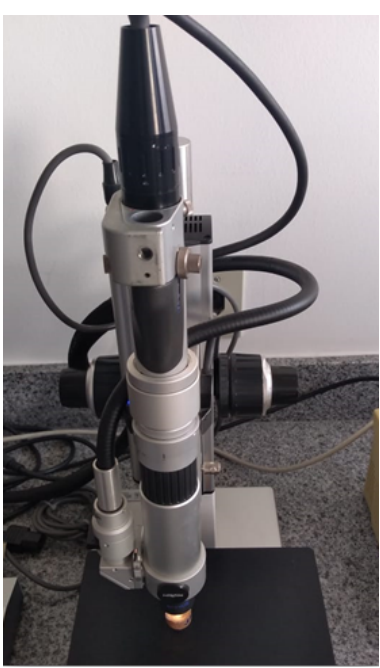

Fonte: Autores.

Os valores médios dos grupos controles e dos 3 grupos testes estudados, foram analisados estatisticamente através do teste de Wilcoxon e do teste de Análise de Variância (ANOVA), com posterior teste de Tukey (HSD) e/ou Dunnett, os quais permitiram a observação intragrupo (antes e depois de cada produto) e intergrupo (análise comparativa dos grupos). A análise de variância foi checada quanto as suas pressuposições estatísticas de normalidade dos resíduos via análise confirmatória gráfica (não apresentada neste estudo) e por meio do teste de normalidade de Shapiro Wilks e de homocedasticidade de Barlet, com valor $\mathrm{p}(\mathrm{p}>0,05)$ evidenciando atendimento dos pressupostos da análise. Todas as análises estatísticas foram realizadas ao nível de 5\% de significância.

\section{Resultados}

As estatísticas descritivas da média, mediana e desvio padrão intragrupo dos valores de Microdureza, relacionando os momentos $\mathrm{T}^{1}$ e $\mathrm{T}^{2}$, respectivamente, antes e depois da aplicação do tratamento clareador e dessensibilizantes, estão descritas na Tabela 1. Em todos os grupos a média de microdureza depois foi menor que a anterior, exceto no G2 e G3, sendo esta última, ligeiramente maior. A mediana depois também se mostrou inferior que a mediana antes em todos os grupos, exceto no G2.

Tabela 1: Microdureza. Estatística descritiva.

\begin{tabular}{|c|c|c|c|c|c|}
\hline & \multicolumn{5}{|c}{ Microdureza } \\
\hline \multirow{2}{*}{ Controle positivo } & T & N & Média & Mediana & Desvio padrão \\
\cline { 2 - 6 } & Antes & 8 & 182,9587 & 234,8500 & 94,22085 \\
\cline { 2 - 6 } & Depois & 8 & 162,6417 & 193,3500 & 90,05693 \\
\hline \multirow{2}{*}{ Controle negativo } & Antes & 8 & 163,5679 & 145,9500 & 124,73759 \\
\cline { 2 - 6 } & Depois & 8 & 143,6083 & 145,4667 & 95,15748 \\
\hline & & & & & \\
\hline
\end{tabular}


Research, Society and Development, v. 10, n. 7, e18810716474, 2021

(CC BY 4.0) | ISSN 2525-3409 | DOI: http://dx.doi.org/10.33448/rsd-v10i7.16474

\begin{tabular}{|c|c|c|c|c|c|}
\hline & Depois & 8 & 235,8125 & 216,7500 & 114,73138 \\
\hline \multirow{2}{*}{$\mathbf{G 2}$} & Antes & 8 & 208,6917 & 176,7667 & 132,68859 \\
\cline { 2 - 6 } & Depois & 8 & 215,3750 & 189,0167 & 135,66263 \\
\hline \multirow{2}{*}{ G3 } & Antes & 8 & 180,6542 & 217,7667 & 93,30704 \\
\hline & Depois & 8 & 181,4917 & 206,5833 & 92,07249 \\
\hline
\end{tabular}

Fonte: Autores.

No que se refere a estatística de teste assinadas por Wilcoxon, tem-se o índice de significância (sig.) por grupo quanto aos valores de microdureza antes e depois (Tabela 2). Nenhum dos grupos apresentou resultados com significância estatística.

Tabela 2: Microdureza. Estatística de teste, teste de classificações assinadas por Wilcoxon.

\begin{tabular}{c|c|c|c|c|c|}
\hline & $\begin{array}{c}\text { Controle positivo } \\
\text { antes - depois }\end{array}$ & $\begin{array}{c}\text { Controle negativo } \\
\text { antes - depois }\end{array}$ & $\begin{array}{c}\text { G1 antes }- \\
\text { depois }\end{array}$ & $\begin{array}{c}\text { G2 antes - } \\
\text { depois }\end{array}$ & $\begin{array}{c}\text { G3 antes - } \\
\text { depois }\end{array}$ \\
\cline { 2 - 6 } & $-1,400^{\mathrm{a}}$ & $-1,540^{\mathrm{a}}$ &,$- 840^{\mathrm{a}}$ &,$- 560^{\mathrm{b}}$ &,$- 420^{\mathrm{b}}$ \\
\hline $\mathbf{Z}$ & 0,161 & 0,123 & 0,401 & 0,575 & 0,674 \\
\hline Sig. & & & & & \\
\hline
\end{tabular}

a. Com base em postos positivos. b. Com base em postos negativos. *. A diferença média é significativa no nível 0.05 . Fonte: Autores.

A microdureza foi analisada intergrupo no período $\mathrm{T}^{2}$ depois da aplicação dos tratamentos clareadores e dessensibilizantes, através do teste de Tukey (honestly significant difference - HSD) (Tabela 3; Figura 7). Não foi observado diferenças de microdureza média entre os grupos, descritivamente houve uma menor microdureza para o grupo controle negativo, enquanto o grupo com o registro de maior microdureza foi o G1.

Tabela 3: Microdureza. Teste de Tukey (HSD).

\begin{tabular}{c|c|c|c|c|c|c|}
\hline Tukey (HSD) & \multicolumn{5}{c}{ Microdureza } & Total \\
\hline Tratamento & $\begin{array}{c}\text { Controle } \\
\text { positivo }\end{array}$ & $\begin{array}{c}\text { Controle } \\
\text { negativo }\end{array}$ & G1 & G2 & G3 & \\
\hline N & 8 & 8 & 8 & 8 & 8 & 40 \\
\hline Médias & 162,6417 & 143,6083 & 235,8125 & 215,3750 & 181,4917 & 187,7858 \\
\hline Sig.* & & & & & & \\
\hline
\end{tabular}

*. A diferença média é significativa no nível 0.05 Fonte: Autores. 
Figura 7: Microdureza. Teste de Tukey (HSD).

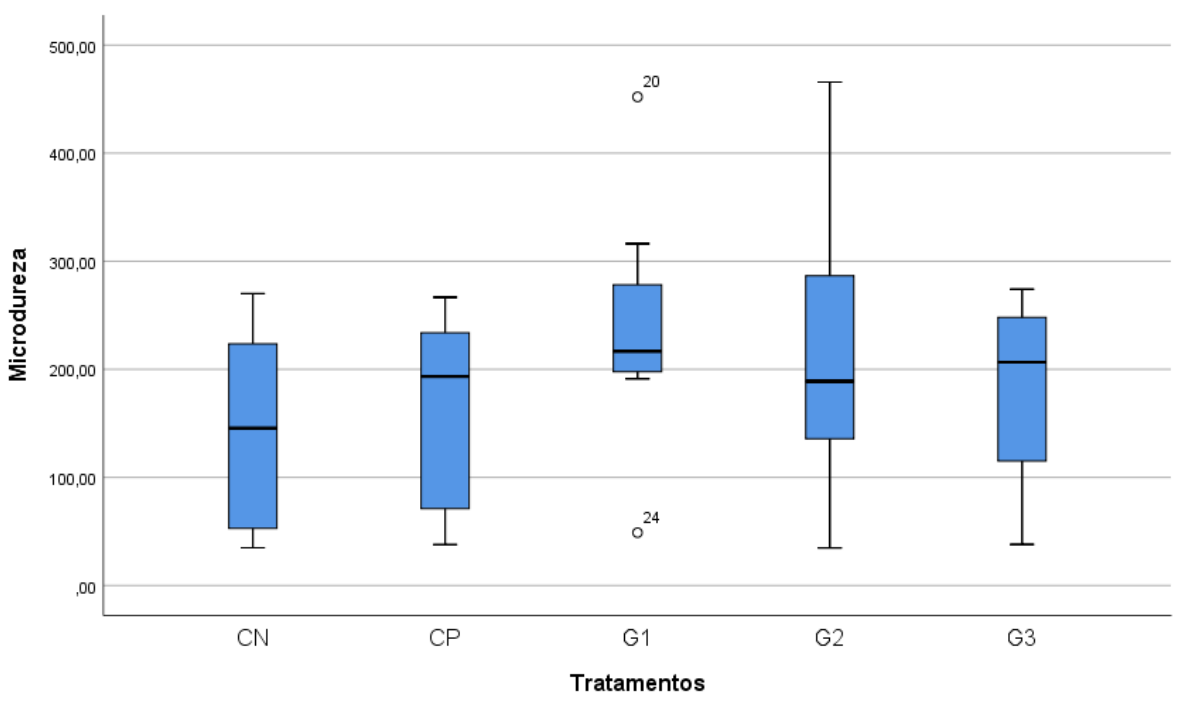

Fonte: Autores.

Em teste de Dunnett foi comparado individualmente o grupo controle positivo com os grupos teste no período $\mathrm{T}^{2}$ (Tabela 4). Não foi observado diferenças significativas entre os grupos.

Tabela 4: Microdureza. Teste de Dunnett, grupo controle positivo comparado a todos os outros grupos.

\begin{tabular}{|c|c|c|c|}
\hline \multirow{5}{*}{$\begin{array}{c}\text { Dunnett t } \\
\text { (>controle)* }\end{array}$} & Tratamento (I) & Tratamento (J) & Diferença média (I-J) - Sig. \\
\hline & Controle Negativo & Controle Positivo & 0,896 \\
\hline & Grupo 1 & Controle Positivo & 0,235 \\
\hline & Grupo 2 & Controle Positivo & 0,384 \\
\hline & Grupo 3 & Controle Positivo & 0,667 \\
\hline
\end{tabular}

*: A diferença média é significativa no nível 0.05 . Fonte: Autores.

Os valores dos ensaios de rugosidade foram submetidos aos mesmos testes estatísticos usados para os valores dos ensaios de microdureza, os testes de Wilcoxon e Tukey (HSD). Para a análise intragrupo, relacionando os momentos $\mathrm{T}^{1}$ e $\mathrm{T}^{2}$, antes e depois da aplicação do tratamento clareador e dessensibilizantes, tem-se a Tabela 5. Todos os grupos apresentaram médias e medianas depois inferiores as iniciais. O grupo que apresentou maior diferença entre as médias $\mathrm{T}^{1}$ e $\mathrm{T}^{2}$ foi o G3, enquanto o grupo com menor variação foi o grupo controle positivo. 
Tabela 5: Rugosidade. Estatística descritiva.

\begin{tabular}{|c|c|c|c|c|c|}
\hline \multicolumn{6}{|c|}{ Rugosidade } \\
\hline & $\mathbf{T}$ & $\mathbf{N}$ & Média & Mediana & Desvio Padrão \\
\hline \multirow{2}{*}{ Controle Positivo } & Antes & 8 & 7,3713 & 7,2450 & 1,02764 \\
\hline & Depois & 8 & 7,0800 & 6,8550 & 1,76469 \\
\hline \multirow{2}{*}{ Controle Negativo } & Antes & 8 & 8,7338 & 8,5600 & 1,71062 \\
\hline & Depois & 8 & 8,1525 & 7,7650 & 1,79570 \\
\hline \multirow{2}{*}{ G1 } & Antes & 8 & 8,6175 & 8,3150 & 1,42682 \\
\hline & Depois & 8 & 8,2838 & 8,1450 & 1,98989 \\
\hline \multirow{2}{*}{ G2 } & Antes & 8 & 10,3988 & 9,5400 & 2,92447 \\
\hline & Depois & 8 & 9,4625 & 8,9350 & 1,99601 \\
\hline \multirow{2}{*}{ G3 } & Antes & 8 & 9,4450 & 8,2550 & 4,29567 \\
\hline & Depois & 8 & 7,5938 & 7,8350 & 2,25532 \\
\hline
\end{tabular}

Fonte: Autores.

A estatística de teste assinadas por Wilcoxon mostra o índice de significância estatística dos ensaios $\mathrm{T}^{1}$ e $\mathrm{T}^{2}$ de rugosidade por grupo (Tabela 6). Nenhum dos grupos apresentou resultados com significância estatística.

Tabela 6: Rugosidade. Estatística de teste, teste de classificações assinadas por Wilcoxon.

\begin{tabular}{|c|c|c|c|c|c|}
\hline & $\begin{array}{c}\text { Controle positivo } \\
\text { antes - depois }\end{array}$ & $\begin{array}{c}\text { Controle negativo } \\
\text { antes - depois }\end{array}$ & $\begin{array}{c}\text { G1 antes - } \\
\text { depois }\end{array}$ & $\begin{array}{c}\text { G2 antes - } \\
\text { depois }\end{array}$ & $\begin{array}{c}\text { G3 antes - } \\
\text { depois }\end{array}$ \\
\hline $\mathbf{Z}$ & $0,000^{\mathrm{a}}$ & $-0,840^{b}$ & $-0,560^{b}$ & $-0,700^{b}$ & $-0,840^{b}$ \\
\hline Sig. (bilateral)* & 1,000 & 0,401 & 0,575 & 0,484 & 0,401 \\
\hline
\end{tabular}

Com base em postos positivos. b. Com base em postos negativos. *A diferença média é significativa no nível 0.05 . Fonte: Autores.

$\mathrm{Na}$ análise intergrupos, realizando a comparação dos grupos quanto a rugosidade superficial média no momento $\mathrm{T}^{2}$ da aplicação dos tratamentos C1 e D1, tem-se a Tabela 7 e o Figura 8. Na Figura 8 apresentam-se algumas observações discrepantes em relação as demais do grupo, porém estas não são estatisticamente influentes ou pontos atípicos. Descritivamente, foi observado diferenças entre os grupos, evidenciando uma menor rugosidade para o grupo controle positivo, enquanto o grupo com o registro de maior rugosidade média foi o G2. 
Tabela 7: Rugosidade. Teste de Tukey (HSD).

\begin{tabular}{|c|c|c|c|c|c|c|}
\hline Tukey (HSD) & & Rugosida & & & & Total \\
\hline Tratamento & Controle positivo & Controle negativo & G1 & $\mathrm{G} 2$ & G3 & --- \\
\hline $\mathbf{N}$ & 8 & 8 & 8 & 8 & 8 & 40 \\
\hline Médias & 7,0800 & 8,1525 & 8,2838 & 9,4625 & 7,5938 & 8,1145 \\
\hline Sig.* & \multicolumn{6}{|c|}{0,134} \\
\hline
\end{tabular}

*. A diferença média é significativa no nível 0.05 Fonte: Autores.

Figura 8: Rugosidade. Teste de Tukey (HSD).

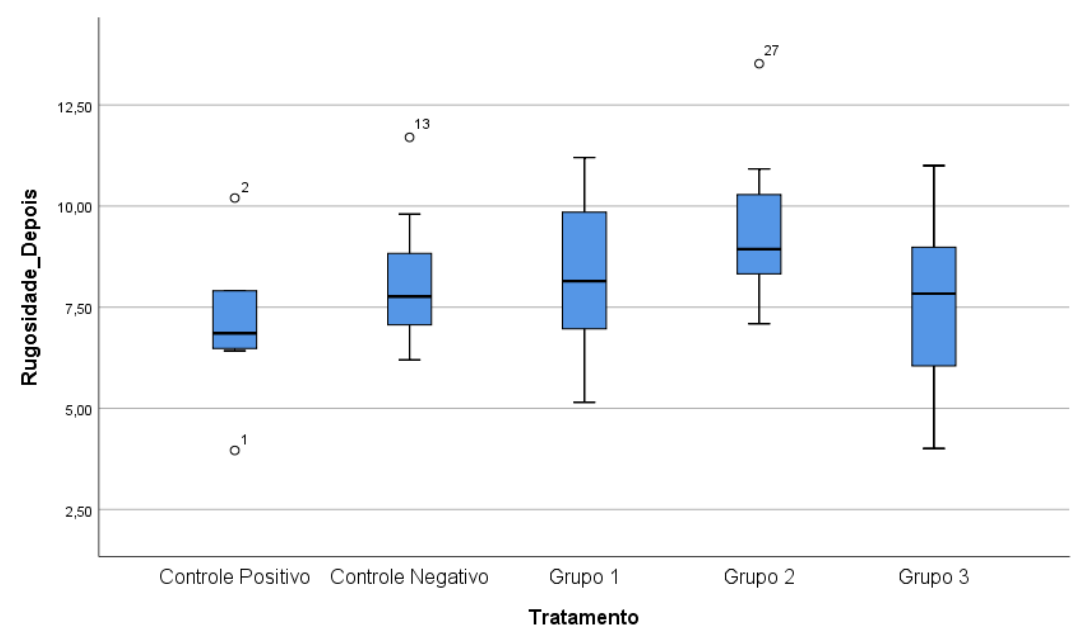

Fonte: Autores.

$\mathrm{Na}$ análise da rugosidade superficial foi possível comparar o grupo controle positivo individualmente com os grupos testes e controle negativo por meio do teste de Dunnett. O grupo controle positivo quando comparado com os demais, no momento $\mathrm{T}^{2}$, apresentou uma diferença significativa em relação ao G2, explicitada na Tabela 8.

Tabela 8: Rugosidade. Teste de Dunnett, grupo controle positivo comparado a todos os outros grupos.

\begin{tabular}{|cccc|}
\hline & Tratamento (I) & Tratamento (J) & Diferença média (I-J) \\
\cline { 2 - 4 } & Controle Negativo & Controle Positivo & 0,340 \\
\cline { 2 - 4 } Dunnett t $(>$ controle) & Grupo 1 & Controle Positivo & 0,287 \\
\cline { 2 - 4 } & Grupo 2 & Controle Positivo & $\mathbf{0 , 0 3 4}$ \\
\hline Grupo 3 & Controle Positivo & 0,593 \\
\hline
\end{tabular}

*: A diferença média é significativa no nível 0.05. Fonte: Autores. 


\section{Discussão}

Os resultados obtidos na pesquisa, nos permite inferir que apenas a utilização de gel dessensibilizante em qualquer momento, antes ou após o clareamento, não foi suficiente para causar diferenças significativas de microdureza e rugosidade na superfície dentária. Foi possível perceber, no entanto, uma tendência após a utilização do agente clareador e do agente dessensibilizante de diminuição das rugosidades.

$\mathrm{Na}$ microdureza, também houve uma tendência a diminuição nos grupos controle positivo, negativo e G1 e de aumento da microdureza média nos grupos G2 e G3. Nos testes de comparação entre grupo controle positivo e demais, para avaliação da rugosidade no momento depois, foi possível perceber que entre os grupos controle positivo e grupo 2 houve uma diferença média considerada significante, em que o grupo 2, tratado com dessensibilizante apenas antes do clareamento, apresentou rugosidade média superior ao controle positivo.

Corroborando com os resultados encontrados nesse estudo, tem-se o trabalho de Torres et al. (2015), o qual, analisou a rugosidade superficial do esmalte dental de dentes bovinos submetidos a tratamento clareador com peróxido de hidrogênio a $35 \%$ e em seguida, a tratamento dessensibilizante com Desensibilize KF 0,2\%. No entanto, diferentemente de Torres et al. (2015), o objetivo foi analisar a ação de dessensibilizantes não apenas no momento posterior ao tratamento clareador, mas, também, no momento anterior a ação deste quando utilizado nos dois momentos, antes e depois. Os resultados encontrados por Torres et al. (2015) também não apresentaram diferenças significativas na rugosidade média do esmalte utilizando o agente dessensibilizante após o clareamento.

China, Souza, Gomes, Alexandrino e Silva (2014) avaliou o efeito do fluoreto de fosfato neutro e acidulado sobre a microdureza e a rugosidade superficial do esmalte clareado utilizando peróxido de hidrogênio a $35 \%$ com e sem adição de cálcio. No que se refere a rugosidade, não foi encontrada nenhuma diferença significativa em qualquer combinação de tratamentos, semelhante aos resultados encontrados nesse estudo. Quanto a microdureza, diferentemente dos resultados dessa pesquisa, o estudo de China et al. (2014) apresentou resultados com diferenças significativas após os tratamentos com fluoreto de fosfato acidulado combinado com peróxido de hidrogênio a $35 \%$ e no clareamento sem fluorterapia complementar, apresentando diminuição na microdureza.

Com relação ao grupo controle negativo, os resultados dessa pesquisa divergem da realizada por Lewinstein, Fuhrer, Churaru e Cardash (2004), visto que, nos dois trabalhos os grupos foram expostos ao clareamento com peróxido de hidrogênio a 35\% e avaliados antes e após a exposição, enquanto que nessa pesquisa não foi observada nenhuma alteração significativa da microdureza, Lewinstein et al. (2004) apresentou reduções significativas da mesma e, também, observaram que a fluorterapia promove a restauração dos tecidos afetados pelo clareamento, o que era esperado para os dessensibilizantes estudados, porém não confirmado por essa pesquisa.

Em um outro estudo também de análise de rugosidade e microdureza do esmalte dentário, exposto a diferentes tipos de agentes clareadores, Pinto, Oliveira, Cavalli e Giannini (2004) corroboram com Lewinstein et al., (2004) tendo em vista apresentaram alterações significativas antes e após a exposição, no entanto, os resultados encontrados pelos autores supracitados contrapõem os resultados de alterações não significantes encontrados nesse estudo. Além de rugosidade e microdureza, foi possível perceber que a ação a agentes clareadores também modificou a morfologia do esmalte superficial, através da análise de microscopia eletrônica de varredura (MEV).

Pintado-Palomino e Tirapelli (2015) em avaliação ao efeito de vários dessensibilizantes associados ao clareamento com peróxido de hidrogênio a $35 \%$ ou peróxido de carbamida a $16 \%$ na rugosidade e microdureza do esmalte e dentina, observaram que a rugosidade do esmalte não foi afetada pelo uso dos agentes clareadores quando estes foram associados aos dessensibilizantes testados, contradizendo com os resultados encontrados nesse estudo, onde houve alteração na rugosidade dos grupos estudados. 
As alterações de rugosidade e de microdureza da superfície do esmalte dentário divergem bastante na literatura, isso pode ser consequência dos diferentes delineamentos experimentais adotados em cada estudo. Outras variáveis como as alterações normais na morfologia do esmalte dos dentes analisados podem ser maiores do que aquelas atribuídas aos efeitos dos peróxidos nos dentes, dificultando a uniformidade da amostra, podendo, assim, afetar os resultados da pesquisa (Torres et al. 2015).

Algumas limitações puderam ser observadas durante a realização dessa pesquisa, por se tratar de um estudo in vitro, as condições bucais não são reproduzidas fielmente, tais como, estruturas anatômicas, potencial hidrogênio-iônico da cavidade oral, e microbiota residente (Freires et al. 2010), o mesmo estudo in situ poderia ser realizado permitindo a análise das mesmas variáveis, no entanto, reproduzindo as condições bucais. Além disso, existe uma gama de agentes dessensibilizantes com diferentes composições que podem, também, ter seus efeitos estudados e comparados em estudos futuros. Assim como, a realização posterior de estudos in vivo analisando a atuação real desses agentes sobre a sintomatologia dolorosa dos pacientes, possibilitando, a determinação de uma conduta de tratamento ideal da sensibilidade dentária pós clareamento, contudo esse estudo respalda o uso dos dessensibilizantes como um tratamento viável para a sensibilidade dentária.

\section{Conclusão}

Com base nos resultados obtidos neste estudo, podemos concluir que a utilização do dessensibilizante estudado, antes e/ou depois do clareamento de consultório, utilizando peróxido de hidrogênio a 35\% não altera significantemente a rugosidade e microdureza de superfície do esmalte dental bovino.

\section{Referências}

Araújo, I. D. T., Sousa, S. K., Neves, P. T. V. O., Costa, M. J. F., Assunção, I. V., \& Borges, B. C. D. (2021). The combined use of systemic analgesic/antiinflammatory drugs and a bioactive topical desensitizer for reduced in-office bleaching sensitivity without jeopardizing the hydrogen peroxide efficacy: a randomized, triple blinded, split-mouth clinical trial. Clinical oral investigations, 10.1007/s00784-021-03948-y. Advance online publication. https://oiorg.ez121.periodicos.capes.gov.br/10.1007/s00784-021-03948-y

Brännström, M., Lindén, L. Å., \& Åström, A. (1967). The hydrodynamics of the dental tubule and of pulp fluid. Caries research, $1(4), 310-317$.

Cerqueira, R. R. D., Hofstaetter, F. L., Rezende, M., Martins, G. C., Loguercio, A. D., Reis, A., \& Kossatz, S. (2013). Efeito do uso de agente dessensibilizante na efetividade do clareamento e na sensibilidade dental. Revista da Associacao Paulista de Cirurgioes Dentistas, 67(1), 64-67.

China, A. L., Souza, N. M., de L Gomes, Y. D. S., Alexandrino, L. D., \& Silva, C. M. (2014). Effect of fluoride gels on microhardness and surface roughness of bleached enamel. The open dentistry journal, 8,188 .

Crepaldi, G. B., \& Marques, J. P. V. (2018). Hipersensibilidade dentinária do diagnóstico ao tratamento: uma revisão de literatura.

Douglas-de-Oliveira, D. W., Paiva, S. M., \& Cota, L. O. M. (2017). Etiologia, epidemiologia e tratamento da hipersensibilidade dentinária: uma revisão de literatura. Braz J Periodontol-December, 27(04).

Freires, I. D. A., Alves, L. A., Jovito, V. D. C., Almeida, L. D. F. D. D., Castro, R. D. D., \& Padilha, W. W. N. (2010). Atividades antibacteriana e antiaderente in vitro de tinturas de Schinus terebinthinfolius (Aroeira) e Solidago microglossa (Arnica) frente a bactérias formadoras do biofilme dentário. Odontologia Clínico-Científica (Online), 9(2), 139-143.

Gillam, D. G. (2013). Current diagnosis of dentin hypersensitivity in the dental office: an overview. Clinical Oral Investigations, 17(1), 21-29.

Gomes, C. S., Filho, J. D. N., Penelas, A. G., \& Fonseca, P. D. S. G. (2015). Avaliação de hipersensibilidade dentinária em função do procedimento clareador: revisão de literatura. Revista Brasileira de Odontologia, 71(2), 194.

Goncalves, C. N., \& Santos, R. C. (2017). Teorias da estimulação dos nociceptores intradentinários em hipersensibilidade dentária.

Hebling, J., Ribeiro, A. P. D., \& Costa, C. A. D. S. (2010). Relação entre materiais dentários e o complexo dentino-pulpar. Robrac, 1-9.

Lewinstein, I., Fuhrer, N., Churaru, N., \& Cardash, H. (2004). Effect of different peroxide bleaching regimens and subsequent fluoridation on the hardness of human enamel and dentin. The Journal of prosthetic dentistry, 92(4), 337-342.

Markowitz, K. (2010). Pretty painful: why does tooth bleaching hurt?. Medical hypotheses, 74(5), 835-840.

Pashley, D. H. (1986). Dentin permeability, dentin sensitivity, and treatment through tubule occlusion. Journal of endodontics, 12(10), 465-474. 
Research, Society and Development, v. 10, n. 7, e18810716474, 2021

(CC BY 4.0) | ISSN 2525-3409 | DOI: http://dx.doi.org/10.33448/rsd-v10i7.16474

Pintado-Palomino, K., Peitl Filho, O., Zanotto, E. D., \& Tirapelli, C. (2015). A clinical, randomized, controlled study on the use of desensitizing agents during tooth bleaching. Journal of dentistry, 43(9), 1099-1105.

Pintado-Palomino, K., \& Tirapelli, C. (2015). The effect of home-use and in-office bleaching treatments combined with experimental desensitizing agents on enamel and dentin. European journal of dentistry, 9(1), 66.

Pinto, C. F., Oliveira, R. D., Cavalli, V., \& Giannini, M. (2004). Peroxide bleaching agent effects on enamel surface microhardness, roughness and morphology. Brazilian oral research, 18(4), 306-311.

Públio, J. C., D'Arce, M. B., Ambrosano, G. M., Aguiar, F. H., Lovadino, J. R., Paulillo, L. A., \& Lima, D. A. (2015). Efficacy of tooth bleaching with the prior application of a desensitizing agent. Journal of investigative and clinical dentistry, 6(2), 133-140.

Rebelo, D., Loureiro, M., Ferreira, P., Paula, A., \& Carrilho, E. (2011). Tratamento médico dentário da hipersensibilidade dentinária-o estado da arte. Revista Portuguesa de Estomatología, Medicina Dentária e Cirugia Maxilofacial, 52(2), 98-106.

Rocha, C. S., Prado, M., Simão, R. A., Lima, C. O., \& Gusman, H. (2016). Efeito de agentes dessensibilizantes na obliteração dos túbulos dentinários-estudo in vitro. Revista Brasileira de Odontologia, 73(4), 272.

Torres, B. M., Quiles, B. T., Guiraldo, R. D., Moura, S. K., Lopes, M. B., \& Berger, S. B. (2015). Efeito de um agente dessensibilizante na rugosidade do esmalte após clareamento. Journal of Health Sciences, 17(2). 